\title{
Resonancia ferromagnética en vidrios metálicos
}

\author{
H.GARCÍA-MIQUEL', J.M.GARCÍA², J.M.GARCÍA-BENEYTEZ ${ }^{2}$, M.VÁZQUEZ ${ }^{2}$, G.KURLYANDSKAYA ${ }^{3}$ \\ ${ }^{1}$ Dpto. Ing. Electrónica E.T.S.I.T. , Univ. Politécnica de Valencia, Camino de Vera s/n, 46022 Valencia, Spain, e-mail: hgmiquel@eln.upv.es \\ 2 Instituto de Magnetismo Aplicado (RENFE-UCM) e Instituto de Ciencia de Materiales (CSIC), Apdo. 155, 28230 Las Rozas(Madrid). \\ ${ }^{3}$ Dpto. Electricidad y Electrónica, Univ. del País Vasco UPV-EHU ${ }^{3}$, Apdo.644, 48080 Bilbao.
}

En el presente trabajo se ha medido la frecuencia de resonancia ferromagnética (FMR) de vidrios metálicos en forma de microhilos de composición $\left(\mathrm{Fe}_{\mathrm{x}} \mathrm{Co}_{1-\mathrm{x}}\right)_{72.5} \mathrm{Si}_{12.5} \mathrm{~B}_{15}$. Estos microhilos constan de un núcleo magnético de unas $5 \mu \mathrm{m}$ de diámetro y una cobertura de vidrio, siendo el diámetro total de entre 10 y $15 \mu \mathrm{m}$ según la muestra. La técnica experimental empleada consiste en la sustitución del dieléctrico de una línea de transmisión coaxial por los microhilos a medir, y en la medida de la absorción de potencia a frecuencias de microondas. Para cada muestra se ha ido variando la intensidad de un campo externo de magnetización, provocando la variación de la frecuencia de resonancia. Con estas medidas se ha conseguido establecer la relación entre campo de magnetización y frecuencia de resonancia, y mediante el análisis de los datos obtenidos y contrastados con el modelo teórico propuesto se ha obtenido información del campo de anisotropía de los microhilos. Finalmente, el campo de anisotropía calculado se ha contrastado con el obtenido mediante la medida del ciclo de histéresis quasi-estático.

Palabras clave: Resonancia ferromagnética, anisotropía magnética, microhilos amorfos, materiales para microondas.

\section{Ferromagnetic Resonance in Metallic Glasses}

In the presently work ferromagnetic resonance (FMR) of metallic glasses in microwires form of composition (FexCo1-x)72.5 Si12.5 B15 has been measured. The ferromagnetic resonance frequency of amorphous magnetic microwires has been obtained from power absorption measurements in the microwave frequency range. The experimental technique here employed consists on the replacement of the dielectric of a coaxial transmission line by the sample to be measured. From the evolution of the resonance frequency with a dc applied magnetic field, the anisotropy field of the microwires has been deduced, and successfully compared to that obtained from quasi-static hysteresis loops.

Key words: Ferromagnetc resonance, magnetic anisotropy, amorphous microwires, microwave materials.

\section{INTRODUCCIÓN}

Los vidrios metálicos son sólidos amorfos conductores que presentan propiedades magnéticas muy interesantes para su aplicación como sensores, colectores de flujo magnético, apantallamiento electromagnético y dispositivos de microondas (1-4). Las muestras a estudiar se encuentran en forma de microhilos con un núcleo magnético de unos $5 \mu \mathrm{m}$ de diámetro y con una cobertura de vidrio, siendo el diámetro total de unas $15 \mu \mathrm{m}$. Las muestras se han fabricado por el método de enfriamiento ultrarrápido de Taylor-Ulitovsky, y de composición $\left(\mathrm{Fe}_{\mathrm{x}} \mathrm{Co}_{1-\mathrm{x}}\right)_{72.5} \mathrm{Si}_{12.5} \mathrm{~B}_{15}$. Las muestras ricas en Fe presentan magnetostricción negativa y las ricas en Co positiva, siendo la magnetostricción nula para $x=5$. Los diferentes coeficientes de dilatación térmica del núcleo metálico y la capa de vidrio originan esfuerzos mecánicos sobre la muestra, provocando la aparición de anisotropía de origen magnetoelástico (5-7). Normalmente se presentan dos zonas, un núcleo central con anisotropía axial, y una corteza con anisotropía radial o circular según la magnetostricción de la muestra.

El objetivo de este estudio es la medida de la frecuencia de Resonancia Ferromagnética, y a partir de estas medidas obtener información al respecto de la anisotropía de origen magnetoelástico de la muestra, según el modelo teórico que relaciona FMR y campo estático aplicado. Se ha medido también el ciclo de histéresis de las muestras, a partir del cual se determina el campo de anisotropía, que se compara satisfactoriamente con el obtenido de las medias de FMR.

\section{MODELO TEÓRICO}

En un material ferromagnético los espines se acoplan para dar como resultado una imanación macroscópica, al menos localmente.

Bajo la aplicación de un campo magnético constante $\mathrm{H}_{\mathrm{o}}$ que sature la muestra, y de un pequeño campo magnético alterno perpendicular con frecuencia en el rango de microondas, se produce un movimiento de precesión de la imanación alrededor de la dirección de aplicación del campo estático. La ecuación de movimiento es:

$$
\frac{d \vec{M}}{d t}=\gamma \mu_{0}(\vec{M} \times \vec{H})
$$

siendo $\mathrm{M}$ la imanación, $\mathrm{H}$ el campo magnético, $\gamma$ la constante giromagnética, y $\mu_{\mathrm{o}}$ la permeabilidad en el vacío.

Teniendo en cuenta los factores de forma de la muestra, así como el campo de anisotropía $\mathrm{H}_{\mathrm{k}}$ de origen magnetoelástico, la solución de la ecuación (1) según [8] proporciona la siguiente expresión para la frecuencia de resonancia $\omega_{0}$ :

$$
\omega_{o}=\gamma \mu_{o}\left[\left(H_{o}+M_{s}\right)\left(H_{o}+\frac{2 K}{\mu_{o} M_{s}}\right)\right]^{1 / 2} \approx \gamma \mu_{o}\left[M_{s}\left(H_{o}+H_{k}\right)\right]^{1 / 2}
$$



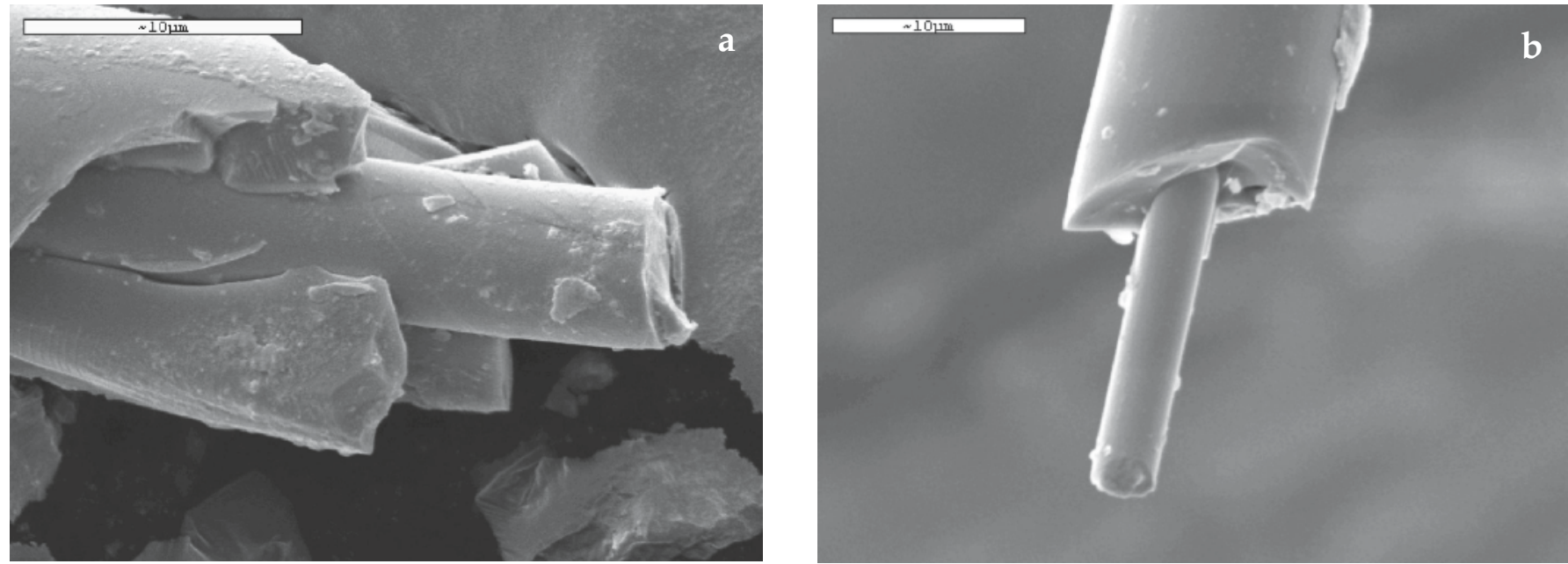

Figura 1: Imágenes obtenidas mediante microscopio electrónico de barrido. Se observa un núcleo que es la parte metálica correspondiente a la aleación, y una cobertura de vidrio que origina esfuerzos termoelásticos sobre el material magnético.

a

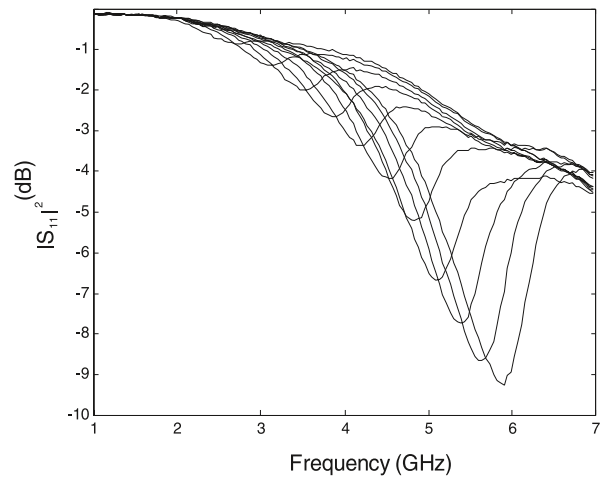

C

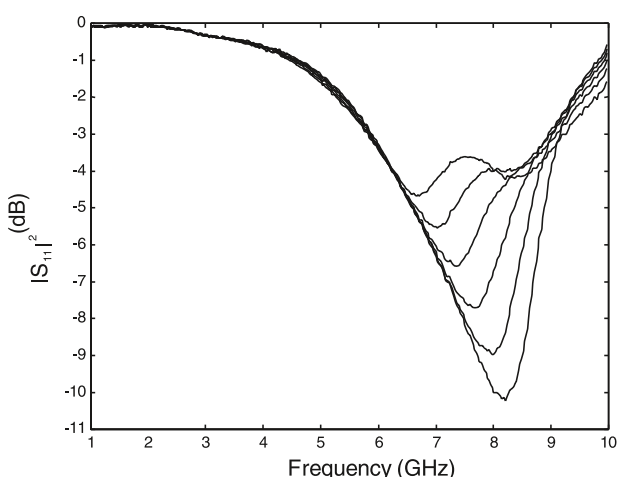

b

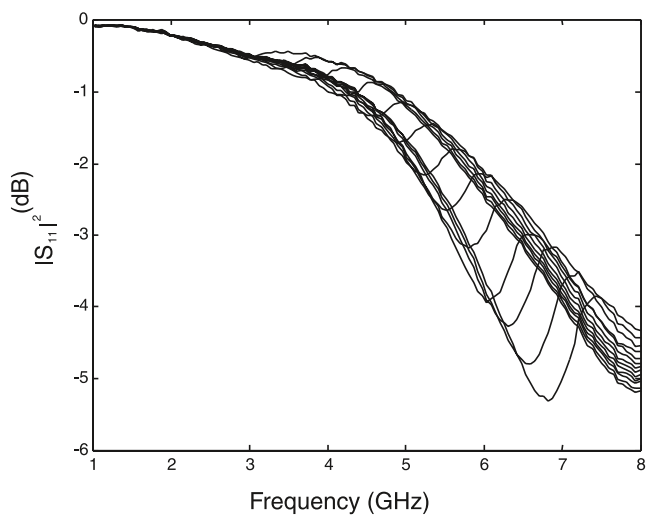

d

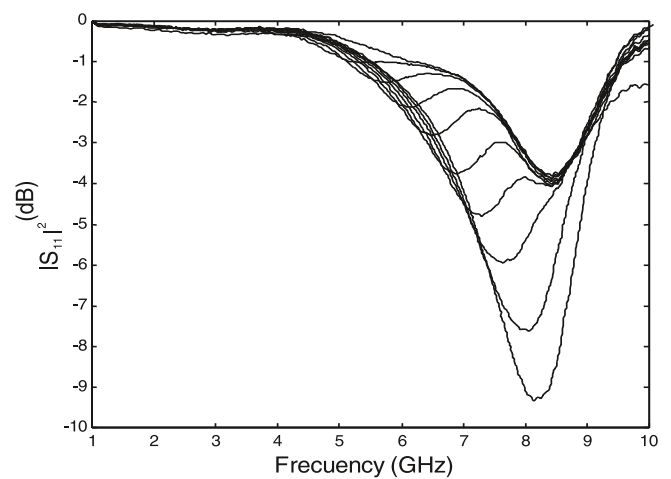

Figura 2: Módulo al cuadrado de $\mathrm{S} 11$ en función de la frecuencia para diferentes valores del campo magnético estático Ho. a) $\mathrm{Co}_{72.5} \mathrm{Si}_{12.5} \mathrm{~B}_{15}$, b) $\left.\left.\left(\mathrm{Fe}_{6} \mathrm{Co}_{94}\right)_{72.5} \mathrm{Si}_{12.5} \mathrm{~B}_{15}, \mathrm{c}\right)\left(\mathrm{Fe}_{50} \mathrm{Co}_{50}\right)_{72.5} \mathrm{Si}_{12.5} \mathrm{~B}_{15}, \mathrm{~d}\right) \mathrm{Fe}_{72.5} \mathrm{Si}_{12.5} \mathrm{~B}_{15}$

\section{PROCEDIMIENTO EXPERIMENTAL}

Se han obtenido imágenes de las muestras mediante microscopio electrónico de barrido (SEM). En la Fig. 1 se pueden observar las dos regiones que componen el microhilo, el núcleo metálico y la cobertura de vidrio.

Las medidas de FMR se han llevado a cabo con el analizador de redes HP8720-B. El portamuestras utilizado consiste en un cable coaxial de microondas RG402, con un corto en un extremo, y donde parte del dieléctrico se ha sustituido por la muestra a medir. Los microhilos se disponen axialmente, de forma que al propagarse un modo TEM por el coaxial, el campo magnético incide perpendicularmente sobre el microhilo. El portamuestras se coloca en el interior de un solenoide, que aplica un campo magnético estático $\mathrm{H}_{\mathrm{o}}$ en la dirección axial de la muestra, y la lleva a saturación. La relación entre potencia reflejada y potencia incidente viene dada por el módulo al cuadrado del parámetro $S_{11}$ que se determina experimentalmente. La frecuencia de resonancia se detecta a través de un pico en la absorción de energía, que se desplaza al variar el campo estático $H_{o}$ (9). La ecuación [2] predice una relación cuadrática entre el campo aplicado y la frecuencia de resonancia. De estas medidas, y mediante la extrapolación para $\mathrm{f}_{0}=0$, se ha obtenido el campo de anisotropía. En la Fig. 2 se observan las medidas de FMR de las muestras estudiadas. En la figura se superponen las gráficas para diferentes valores de campo estático de magnetización 


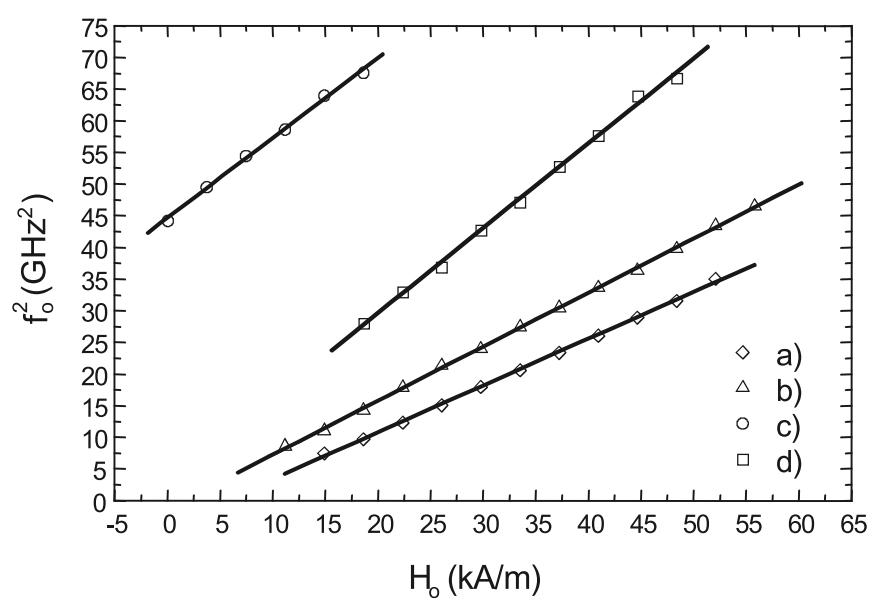

Figura 3: Dependencia de la frecuencia de resonancia al cuadrado con el campo estático Ho. a) $\mathrm{Co}_{72.5} \mathrm{Si}_{12.5} \mathrm{~B}_{15}$, b) $\left(\mathrm{Fe}_{6} \mathrm{Co}_{94}\right)_{72.5} \mathrm{Si}_{12.5}$ $\left.\left.\mathrm{B}_{15}, \mathrm{c}\right)\left(\mathrm{Fe}_{50} \mathrm{Co}_{50}\right)_{72.5} \mathrm{Si}_{12.5} \mathrm{~B}_{15}, \mathrm{~d}\right) \mathrm{Fe}_{72.5} \mathrm{Si}_{12.5} \mathrm{~B}_{15}$

TABla 1: CAMPO DE ANISOTROPÍA DEDUCIDO DE LAS MEDIAS DE FMR Y DE LOS CICLOS DE HISTÉRESIS PARA CADA UNA DE LAS MUESTRAS ESTUDIADAS.

\begin{tabular}{|c|c|c|}
\hline Muestra / Método & FMR & Ciclo de histéresis \\
\hline $\mathrm{Co}_{72.5} \mathrm{Si}_{12.5} \mathrm{~B}_{15}$ & $\mathrm{H}_{\mathrm{k}}=-5.4 \mathrm{kA} / \mathrm{m}$ & $\left|\mathrm{H}_{\mathrm{k}}\right|=7.4 \mathrm{kA} / \mathrm{m}$ \\
\hline$\left(\mathrm{Fe}_{6} \mathrm{Co}_{94}\right)_{72.5} \mathrm{Si}_{12.5} \mathrm{~B}_{15}$ & $\mathrm{H}_{\mathrm{k}}=-1.5 \mathrm{kA} / \mathrm{m}$ & $\left|\mathrm{H}_{\mathrm{k}}\right|=0.5 \mathrm{kA} / \mathrm{m}$ \\
\hline$\left(\mathrm{Fe}_{50} \mathrm{Co}_{50}\right)_{72.5} \mathrm{Si}_{12.5} \mathrm{~B}_{15}$ & $\mathrm{H}_{\mathrm{k}}=35.4 \mathrm{kA} / \mathrm{m}$ & - \\
\hline $\mathrm{Fe}_{72.5} \mathrm{Si}_{12.5} \mathrm{~B}_{15}$ & $\mathrm{H}_{\mathrm{k}}=2.1 \mathrm{kA} / \mathrm{m}$ & $\left|\mathrm{H}_{\mathrm{k}}\right|=2 \mathrm{kA} / \mathrm{m}$ \\
\hline
\end{tabular}

$\mathrm{H}_{\mathrm{o}}$. En la Fig. 3 se muestran las gráficas de las cuatro muestras medidas que relacionan campo estático $\mathrm{H}_{\mathrm{o}}$ con frecuencia de resonancia al cuadrado, en concordancia con el modelo teórico identificado por la ecuación [2].

Se ha medido el ciclo de histéresis quasi-estático de la muestra utilizando un magnetómetro de masa vibrante (VSM), obteniéndose el campo de anisotropía. En la Fig. 4 se muestran los ciclos de histéresis de dos de las muestras, de donde se deduce el campo de anisotropía.

\section{RESULTADOS}

Los resultados experimentales que relacionan frecuencia de resonancia al cuadrado frente a campo magnético estático, presentan una relación lineal que concuerda con la modelización teórica expresada en al ecuación [2]. De la extrapolación de las medidas para $\mathrm{f}_{0}=0$ obtenemos los campos de anisotropía. En la tabla I, se muestran los campos de anisotropía para cada muestra, obtenidos de las medidas de FMR y de los ciclos de histéresis. Según la ecuación [2], las muestras con 50\% y $100 \%$ de Fe presentan campo de anisotropía positivo, lo cual indica que obtendríamos una frecuencia de resonancia ferromagnética incluso con $\mathrm{H}_{0}=0$. El ciclo de histéresis de la muestra $\left(\mathrm{Fe}_{50} \mathrm{Co}_{50}\right)_{72.5} \mathrm{Si}_{12.5} \mathrm{~B}_{15}$ muestra un ciclo casi rectangular y con una remanencia prácticamente igual a la magnetización de saturación. Esto indica que la anisotropía no es transversal como en las demás muestras, sino que es longitudinal.
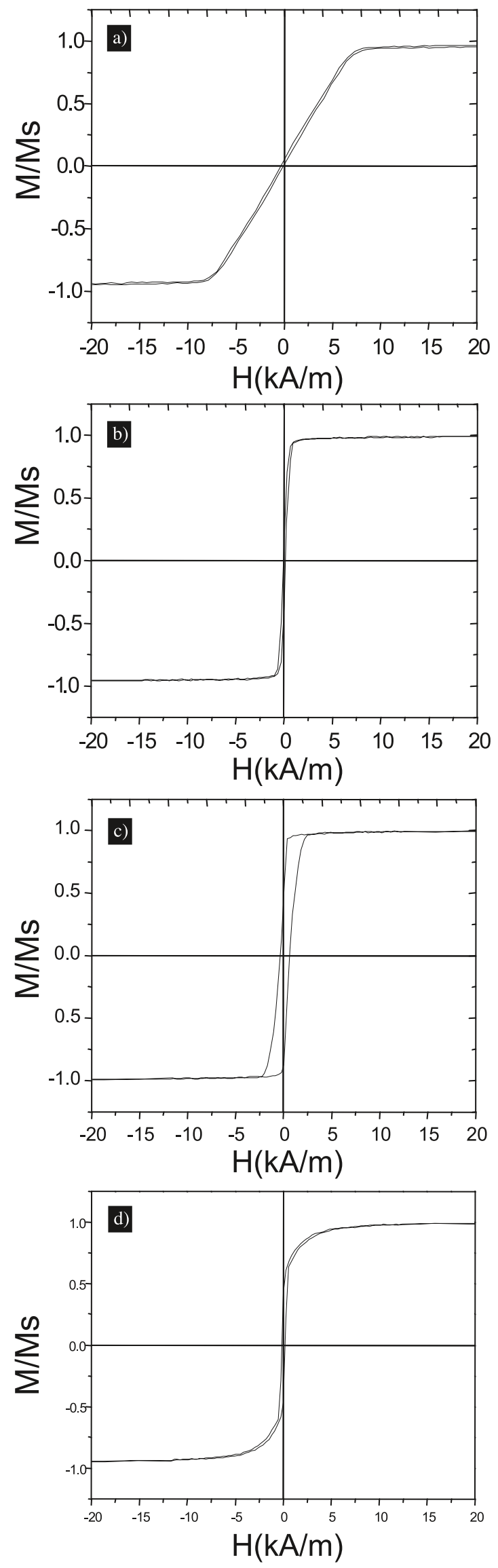

Figura 4: Ciclos de histéresis obtenidos mediante magnetómetro de masa vibrante. a) $\mathrm{Co}_{72.5} \mathrm{Si}_{12.5} \mathrm{~B}_{15}$, b) $\left(\mathrm{Fe}_{6} \mathrm{Co}_{94}\right)_{72.5} \mathrm{Si}_{12.5} \mathrm{~B}_{15}$,

c) $\left.\left(\mathrm{Fe}_{50} \mathrm{Co}_{50}\right)_{72.5} \mathrm{Si}_{12.5} \mathrm{~B}_{15}, \mathrm{~d}\right) \mathrm{Fe}_{72.5} \mathrm{Si}_{12.5} \mathrm{~B}_{15}$ 


\section{CONCLUSIONES}

El método basado en la medida del parámetro $S_{11}$ de una línea coaxial cuyo dieléctrico ha sido sustituido por los microhilos colocados axialmente, y con un corto en un extremo, se presenta como un método efectivo que permite medir la frecuencia de resonancia ferromagnética. La relación entre FMR y campo estático se mide experimentalmente y se compara satisfactoriamente con el modelo teórico propuesto. De las medias de FMR se ha deducido el campo de anisotropía de las muestras y se ha comparado satisfactoriamente con el obtenido a través de los ciclos de histéresis.

\section{BIBLIOGRAFÍA}

1. A. Antonov, A. Granovski, A. Lagar'kov, N. Perov, N. Usov and T. Furmanova, "The features of GMI effect in amorphous wires at microwaves", Physica A, vol. 241, pp. 420-424, (1997).

2. P. M. Jacquart and O. Acher, "Permeability Measurement on Composites
Made of Oriented Metallic Wires from 0.1 to $18 \mathrm{GHz}$, IEEE Trans. Microwave Th. Tech., vol. 44, no 11, pp. 2116-2120, (1996).

3. O. Ishii, M. Senda, K. Takei, Y. Koshimoto and T. Mori, "Application of Co-based amorphous ribbon to a noise filter and a shielded cable", J. Appl. Phys., vol. 79, no 8, pp. 5483-5485, (1996).

4. O. Acher, P. M. Jacquart, J. M. Fontaine, P. Baclet and G. Perrin, "High Impedance Anisotropic Composites Manufactured from Ferromagnetic Thin Films for Microwave Applications", IEEE Trans. Magn., vol. 30, nº 6, pp 4533-4535, (1994).

5. H. Chiriac, T. A. Ovari and Gh. Pop, "Internal stress distribution in glasscovered amorphous magnetic wires", Phys. Rev. B, vol. 52, n 14, pp. 1010410113, (1995).

6. H. Chiriac, Gh.Pop, T.-A.Ovari and F.Barariu, "Magnetic behaviour of negative and nearly zero magnetostrictive glass-covered amorphous wires", IEEE Trans. Magn. vol. 32, nº 5, pp. 4872-4874, (1996).

7. J. Velazquez, M. Vazquez and A. P. Zhukov, "Magnetoelastic anisotropy distribution in glass-coated microwires", J. Mater. Res., vol. 11,n 10, pp. 24992505, (1996).

8. C. Kittel, "On the Theory of Ferromagnetic Resonance Absorption", Phys. Rev., vol 73, n 2, pp. 155-161, (1948).

9. H. García-Miquel, M.J. Esbrí, J.M. Andrés, J.M. García, J.M. García-Beneytez and M. Vázquez "Power Absorption And Ferromagnetic Resonance In Co-Rich Metallic Glasses", IEEE Trans. Magn. (submitted). 